\title{
Design and Implementation of On-site Testing Module of College English General Testing System
}

\author{
Shiting Dong ${ }^{1,}$ a , Jiahui $\mathrm{Qi}^{1}$, Yan $\mathrm{Ye}^{1}$, Min $\mathrm{Wu}^{1,2}$ \\ ${ }^{1}$ Lab of Modern Educational Technology, University of Science and Technology of China, Hefei, \\ 230026, China; \\ ${ }^{2}$ School of Software Engineering, University of Science and Technology of China, Hefei, 230026, \\ China. \\ a18919904823@163.com
}

Keywords: Testing System, Testing Questions Structure, Componentized, On-Site Testing.

\begin{abstract}
According to the deficiencies of on-site testing module of current testing system, such as fixed types, complicated operations, poor user experience etc. After detailed requirements analysis, the author defined easily extensible structure of testing questions, designed new architecture of system, developed on-site testing module of online testing system that used in current college. In the process of development, the author added modular design and componentized ideology in order for improving efficiency of development, reducing the cost of system expansion and maintenance, meanwhile performance of system was improved by using engineering tools such as module package.
\end{abstract}

\section{Introduction}

Online Testing is a form of exam that not via traditional paper, but use the advantages of local area network, let participants manipulate computer to exam on network. Compared to the traditional paper exam, online testing system simplify tedious examination procedures, save the cost of human and material, provide convenient and automatic way for statistics and management.

In this paper, at first, the author analyze current situation and deficiencies of domestic and foreign testing system, especially the on-site testing module is analyzed in detail for improvement, and then analyze requirements of college English general testing system(hereinafter referred to as "the general testing system"), next design system architecture, especially design and develop on-site testing module, at last complete corresponding summary, the author hope this system can provide reference for development of after system.

\section{Current Situation and Deficiencies of Domestic and Foreign Testing System}

\subsection{Current Situation of Domestic and Foreign System.}

The existing testing systems are primary used for international certification exam, including language proficiency test and special skills certification. The United States as the first country to using, launched a series of testing systems in order to certificating level of English, those systems are used widely as the computer-assisted language testing system, such as GRE, TOEFL, GMAT etc. At the beginning of 21 st century, with the rise of modern network education in our country, many colleges start to use testing system that based on architecture of $\mathrm{C} / \mathrm{S}$ or $\mathrm{B} / \mathrm{S}$, such as computer grade examination system, and college English audio-visual intelligence testing platform.

In recent years, the above common domestic and foreign testing systems begin to gradually change from local application to Web application, however, there are still a lot of deficiencies in versatility, system performance and user experiences, those need further research.

\subsection{Deficiencies of the Existing Testing System.}

Although the existing testing systems have been gradually improved, the author still found systems have issues of poor extension, single testing pattern, strong target and other issues, mainly show for the following: Because complex process, the setting questions module only can be operated by specific 
person. The method of saving testing questions is fixed, the types of testing questions can't be change, and testing paper can't be extended. There are two patterns of existing testing systems, one is strict on-site testing pattern and another is total practice pattern, each only supports the respective testing pattern, resulting testing pattern is single.

On-siting testing module is core of testing system, there are following deficiencies after survey:

(1) Unfriendly interface. The testing paper interface of existing testing systems reference typesetting format of traditional paper, ignore the problem that the paper content is showed limitedly on computer full screen, taking notes is inconvenient on computer.

(2) Complex answer operations. Now the operation is mature only for simple selected question, but for blanks and other need candidates to roll mouse for checking carefully to ensure answer is correct.

(3) Mechanism for handing emergency is not perfect, performance issues is not enough attention. There are some common performance issues, such as loading test paper is slow, response of operating need a long time, multimedia elements are loaded lazily etc.

In view of above deficiencies, developers need to start from the view of product, develop a testing system with generality, easy to expend, good user experience.

\section{Requirements Analysis}

References Requirement engineering is a continuous activity which spans the entire cycle of software development production. The general testing system designed by author is a product used in colleges and universities, the research object is teachers and students in author's school (University of Science and Technology of China). Through the method of investigation, literature research and theory with practice, the author get system module, the main function of module and the system stakeholders, these are shown in Figure 1.

stakeholders

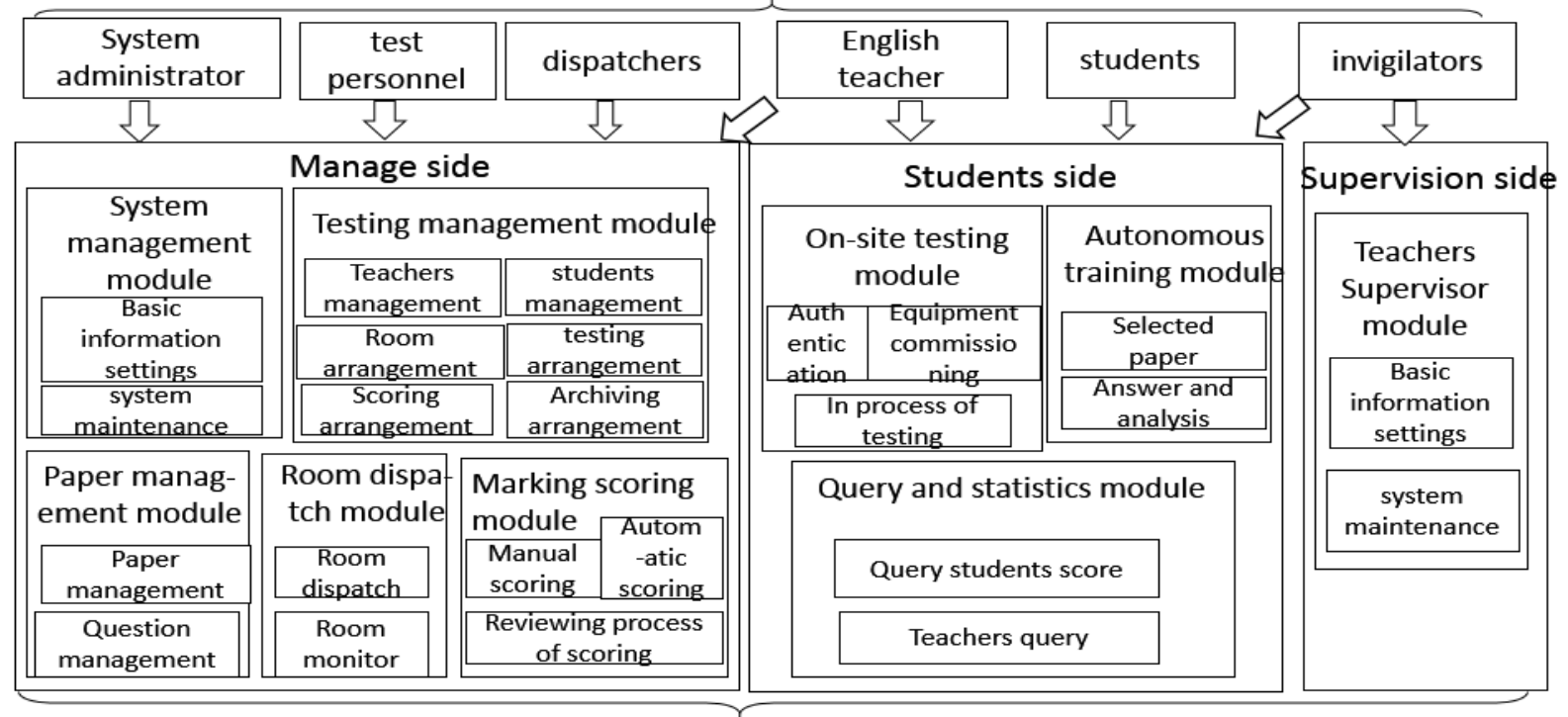

Function module

Fig. 1 Stakeholders of system and functional module

From figure 1, we can see that the system is mainly used by system administrators, test manager, exam dispatchers, invigilators, English teachers and students, the system is divided into management side, supervision side and student side. This paper mainly focuses on the strict on-site testing module (hereinafter referred to as the "on-site testing"), which is controlled by the teachers, the students start the test at the same time. The following figure 2 is the on-site testing activity chart.

The functional differences of existing testing systems is not the main problem the developer face, but scalability, user experience and other nonfunctional indicators have become a key factor to measure the quality of a testing system. Through the analysis, the author get non-functional requirements as follows: rich interface elements, simple answer operation, easy extension of functions, better system performance and so on. 


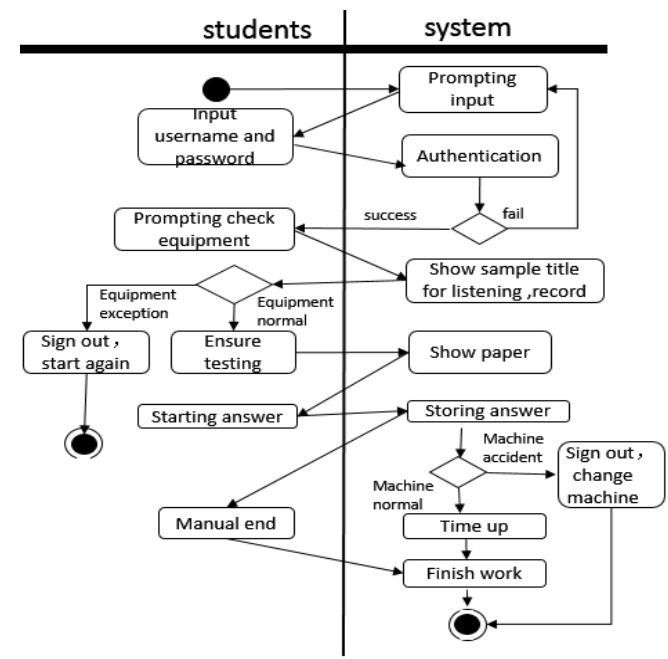

Fig. 2 The activity diagram of on-site testing

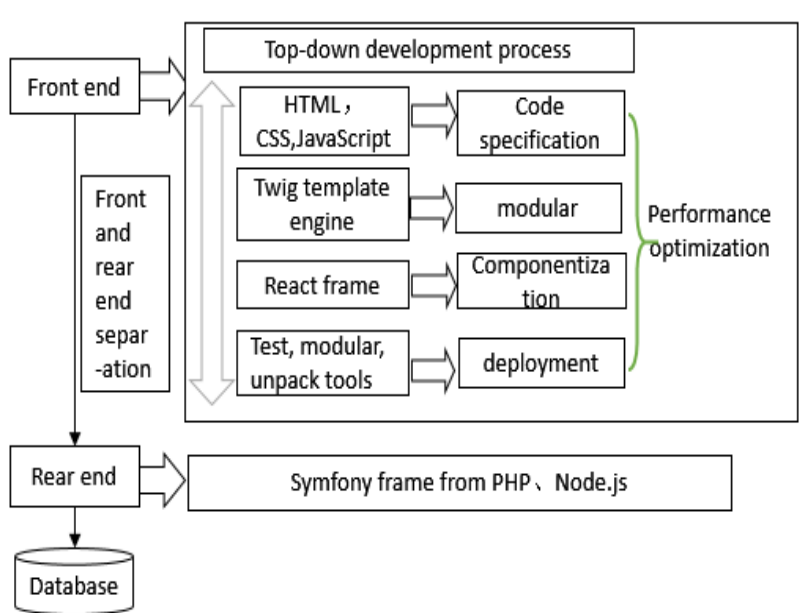

Fig. 3 Architecture of system development

\section{Design and Development of On-Site Sting Module}

\subsection{System Architecture of Development.}

This project is a team development and need to maintain follow-up, so during the process of development to format engineering process has lots of advantages, such as improving speed of development, new people easy to join, high reuse rate of code, easy extension of functions etc. Because development process are based on perfect architecture of system, so the author design architecture diagram of system as figure 3.

From figure 3 , we can see the entire system use the ideology that front end and rear end are separation, database use MySQL, rear end use node.js and Symfony framework from PHP. Front end as the nearest part to users, is core of on-site testing. On the basis of structure layer, presentation layer, behavioral layer, front end joined template engine of Twig, React componentized framework and tools of modular packaging such as Webpack, all of this are used for improving reuse rates of code, increasing extension of system function and robustness of system.

\subsection{Design and Realization of Core Function of On-Site Testing}

In view of on-site testing of the existing systems has so many deficiencies, the author does some improvements in this test system, there are some focus and difficulty in the process of realization:

(1) The structure of testing question and testing paper. The fundamental reason of lacing of versatility in the existing testing systems is fixed attributes of testing question, because testing questions are stored by setting the attributes as the field of database, so deletion and expansion of attributes is inconvenient. In this system, testing questions and papers are described through JSON data format which is convenient to deletion and expansion, let JSON structure as one field of database. Through decomposition, refinement and abstraction for structure of English testing questions, the author get structure of testing questions as following:

\{"stem": "subject", "show-stem-length": if length of subject is shown,

"Flow able": if test question is flow,

"Shuffle": if testing questions options is order,

"show-options-order-num": if testing questions options number is shown,

"Options": ["array of testing questions options "],

"questions-num-limit": if quantities is limited, "pre-show": if advance show,

"Questions": [\{"type": "type of small question", "stem": "subject of small question",

"Options": "options of small question ", "strict": if strict match with correct answer,

"reference-answer":" reference answer ", "answer-analysis": "answer analysis"

\}] $\quad$;

The structure all has nine fields, flowable question is that needing to according to determine process to complete testing questions display and answer, the time used is controllable, such as listening 
questions. This structure not only expend type of questions easily, but also prevent fraud through upset the order of questions and options.

According to abstraction for existing paper structure, the author get structure of paper as following: \{"question-option-shuffle": if questions is order,

"Groups":[\{ "duration": "total time of group ",

"Parts": [ "name": "part name",

"flowable": if this part is flowable question,"duration": "total time of part", "Sections": [ \{“direction": "subject", "shuffle": "if item in section is order ", "Items": []

\section{\}] \}] \}] \}}

We can know from structure, testing paper is consisted by different group, each group is showed independently and has independent time. Group is consisted by one or more parts, the significance of part is distinguishing types of testing questions, item is the minimum unit of paper. This structure of paper can show paper through different types of question, countdown through respective group.

(2) Paper display and answer operation. Because the existing testing systems show paper through static page composed by HTML elements, then add interaction event, resulting many repeats of codes, so this system use development ideology that componentization of React, show testing paper through dynamic generating DOM elements, and through reuse component to reduce the repeat of codes.

After the survey, the author found that commonly used types of questions has 36 species. There are 8 basic interactive types after refinement and abstraction: single choice, multiple choice, fill in blank, determine, short answer, recording, sort, change wrong, let these basic types as basic component, other component are nesting and combination of basic component. The following is component of fill in blank questions written by React:

var Blank Filling $=$ React. Create Class $(\{$ Handle Change: function (event) $\}$

var question_id = this. Props. Question Id; var new Value = event. Target. Value;

Answer [question_id] = new Value; local Storage. Set Item ('answer', JSON. Stringify (answer)) ; ,

Render: function () \{var question _id = this. Props. Question ID; var question_answer $=$ answer [question_id] || "; var answer_area=<input type="text" placeholder="please input answer" default Value $=\{$ question_answer $\}$ on Change $=\{$ this .handle Change $\} />$;

return ( <div class Name="answer-area"> $\{$ answer area $\}</$ div $>$ ) $\} \quad\})$;

We can see the component accept parameter, finish interface of input box, interactive operation of answer, save the answer in local browser and other function.

The component of paper correspond JOSN structure of paper. First, paper component refer group component, then group refer part component, and so on, the outermost layer is paper component, it is shown in specified DOM element by render function of React:

React DOM. Render (<Paper paper= \{paper $\} />$, document .get Element by Id ('content'));

It can be seen that the componentized method is easy for function to expand and maintain.

In addition, this system do certain improvement in aesthetic of interface, simplicity of operation and other use experience: providing multiple columns to display paper, for some questions, the article and question are shown in left and right; Increasing function of taking notes, such as bold and italics and meanwhile, providing text area for recording conveniently, simplify operation at answer, such as, for fill in blank questions, students can answer directly on questions, for some selected questions, prompting options when mouse over. The final paper interface implemented is as Fig. 4 and Fig.5.

(3)Improving robustness of on-site testing. Although performance of software and hardware are always improving, we still can't avoid accidents in process of testing. After survey the author found that the key strategy to processing possible problems of software and hardware is stored and restored of answers. This system finish local storage of answer by localStorage belongs to HTML5 at first, then update the contents of server regularly through asynchronous upload of Ajax. LocalStorage is one of way for persistence local storage, the stored data will not lost when closed browse. 


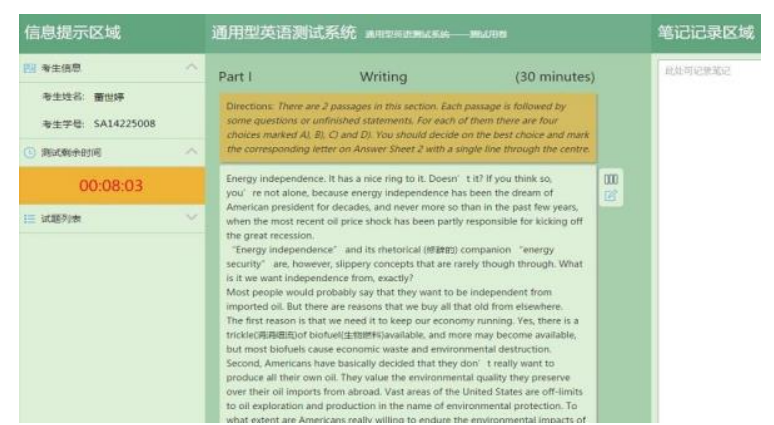

Fig. 4 The testing paper of on-site testing.

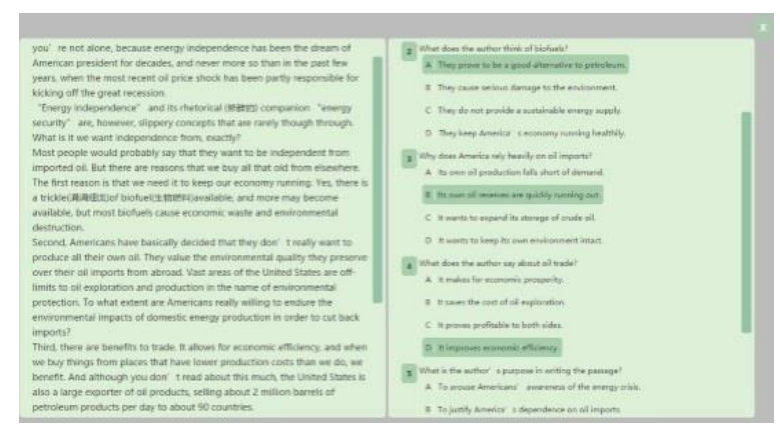

Fig.5 The columns of testing paper

Restoration is paper reverts to the state before the exception after re-login when occur accident during the testing, such as software crashes. In this system, restoration distinguish processing questions and non-processing questions, for non-processing questions, the answer is automatically filled for completed questions through getItem function of localStorage, according to reserved time to restore countdown. For processing questions, one need to restore answers and countdown, other need to restore the progress of the questions, such as listening questions, this realized by save number of group, part, item and already used time of item.

(4) Optimizing performance of system. Performance of system is important factor to influence user experience, it is also the direction of efforts by developer. In this system, the author use the frame of React to improve speed of rendering testing paper, because virtual DOM mechanism from React ensure the paper need to render again only when structure of DOM tree changed, and complexity of algorithm which judging if the structure of DOM tree is changed is only $\mathrm{O}(\mathrm{n})$, so React can improve speed of rendering testing paper. What's more, the author use Webpack let files in system compress and unpack. Webpack is a type of module packer which regard the picture, files of JavaScript, files of CSS and other resource as a module, modules are referred to each other and loaded by demand. Webpack can find all dependent files and then these files can be packed to become one file of JavaScript, through this, system can reduce quantity of requesting resources, so improve the speed of access. Through the above ways, the author enhance the performance of system.

\section{Summary}

This paper in view of deficiencies that on-site testing of the existing testing system have, based on the general testing system version 1.0, combine with actual requirements from current teaching English, design new architecture, join ideology of componentization and modular, use new technical tools such as React, HTML5 and Webpack to complete corresponding improvements, finally, realize on-site testing module of general testing system. But there are still some defect, this system is for strict on-site testing module, lack for personal practicing system, so the next work consider to develop autonomous training subsystem for students, which can make a customized learning plan for different users, provide targeted test for students to practice.

\section{References}

[1]. Zouhong. Design and Realization of Online English Testing System Based on Web. ChengDu: University of Electronic Science and Technology of China. 2012

[2]. Xuxu Liu. Design And Implementation of Online Test System Basic on B/S. modern computer 2010, p.190-193.

[3]. Jianhua Gong.The Application of JSON Data Format in Web Development. Office Automation.2013, p.46-48

[4]. Zhikui Li. Test System Research and Implementation of Network Universal Basic on B/S Mode. Zhejiang University of Technology.2009. 